Article

\title{
Thermo-Responsive Injectable MPEG-Polyester Diblock Copolymers for Sustained Drug Release
}

\author{
Hoon Hyun ${ }^{1, \dagger}$, Seung Hun Park ${ }^{1, \dagger}$, Doo Yeon Kwon ${ }^{1}$, Gilson Khang ${ }^{2}$, Hai Bang Lee ${ }^{1}$ and \\ Moon Suk Kim 1,*
}

1 Department of Molecular Science and Technology, Ajou University, Suwon 443-749, Korea; E-Mails: hhyun79@gmail.com (H.H.); hpt88@ajou.ac.kr (S.H.P.); kdy@ajou.ac.kr (D.Y.K.); hblee@ajou.ac.kr (H.B.L.)

2 Department of BIN Fusion Technology, Chonbuk National University, Jeonju 561-756, Korea; E-Mail: gskhang@jbnu.ac.kr

$\dagger$ These authors contributed equally to this work.

* Author to whom correspondence should be addressed; E-Mail: moonskim@ajou.ac.kr; Tel.: +82-31-219-2608; Fax: +82-31-219-3931.

External Editor: Hsing-Lin Wang

Received: 16 September 2014; in revised form: 7 October 2014 / Accepted: 17 October 2014 / Published: 23 October 2014

Abstract: Thermo-responsive diblock copolymers composed of hydrophilic methoxy poly(ethylene glycol) (MPEG) and hydrophobic biodegradable polyesters were prepared for application as injectable drug delivery systems, because they show a thermo-responsive sol-to-gel transition, especially around body temperature, when dispersed in aqueous solutions. The thermogelling hydrogels formed by hydrophobic aggregation could be varied by changing the components of the hydrophobic polyester part. For the polyester block in the present study, $95 \mathrm{~mol} \%$ of $\varepsilon$-caprolactone (CL) was used for the main polyester chain and $5 \mathrm{~mol} \%$ of $p$-dioxanone (DO) was copolymerized randomly by the MPEG initiator in the presence of $\mathrm{HCl}$ as the catalyst. By adding a small portion of DO into the poly $\varepsilon$-caprolactone (PCL) chains, the temperature range of gelation, the intensity of viscosity and the drug release behavior were changed. The MPEG- $b$-poly( $\varepsilon$-caprolactone-ran-p-dioxanone) (MPEG- $b$-PCDO) hydrogel showed the enhanced drug release in vitro and in vivo compared to MPEG- $b$-PCL hydrogel. Therefore, MPEG-polyester hydrogels may serve as minimally invasive and therapeutic, injectable 
hydrogel systems with adjustable temperature-responsive and biodegradable windows, as well as sustained release of drugs over a certain time period.

Keywords: thermo-responsive hydrogels; biodegradable polyesters; block copolymers; sustained release

\section{Introduction}

Thermo-responsive hydrogels have been developed for the delivery of injectable drugs because: (i) the formulation with drugs is relatively easy; and (ii) the drug delivery can be localized and prolonged [1-5]. Thermo-responsive polymers can be composed of amphiphilic block copolymers. If dispersed in aqueous solutions, they initially assemble into micelles and form "bridged" networks of micelles. These bridged micelles undergo arranged packing triggered by temperature changes to form a macroscopic gel. Thus, the sol-to-gel or gel-to-sol phase transitions are affected by the following conditions: concentration of amphiphilic block copolymer, di-, tri- or multi-block structures, the molecular weight (MW) of block components and the interactions of hydrophobic segments (e.g., hydrophobic interactions, crystallinity, stereocomplexation) [6-11]. Commercially available poloxamers and pluronics comprising poly(ethylene oxide) (PEO) and poly(propylene oxide) (PPO) are representatives of thermo-responsive hydrogels. However, they have several drawbacks for easy clinical applications, such as poor mechanical properties, high permeability, short persistence time and non-biodegradability [12,13]. As reported by several research groups, the PPO blocks of thermo-responsive hydrogels have been replaced with biodegradable polyester blocks to overcome these limitations. In particular, the aliphatic polyester groups, such as polyglycolide (PGA), polylactic acid (PLA) and poly $\varepsilon$-caprolactone (PCL), have been studied with regard to the preparation of thermosensitive hydrogels and tissue engineering scaffolds, due to their excellent biodegradability and biocompatibility [14-18].

During the last decade, our group has reported a thermo-responsive hydrogel comprising methoxy polyethylene glycol (MPEG) and PCL (MPEG- $b$-PCL) that showed a sol-to-gel phase transition at body temperature when dispersed in aqueous solutions at certain concentrations. In various studies, the MPEG- $b$-PCL diblock copolymer has been used as an in vivo carrier for the delivery of protein drugs and as a scaffold for cell growth [19-24]. The MPEG-b-PCL diblock copolymer was shown to be a good candidate for injectable thermo-responsive hydrogels, and therefore, several approaches to modify the MPEG- $b$-PCL diblock copolymer have been employed for more controllable delivery of drugs [25].

To prepare diverse thermo-responsive hydrogels, we reported that the incorporation of L-lactide (LA), $p$-dioxanone (DO) and trimethylene carbonate (TMC) into the PCL block can be accomplished with PCL in various ratios and that thermosensitive gelation could be maintained at body temperature. Random copolymers using CL, LA, DO and TMC are more favorable than PLA and poly(lactic-coglycolic acid) PLGA copolymers if used for biomedical applications, because their degradation does not result in an acidic environment. However, PLA and PLGA copolymers in biological conditions are degraded rapidly and release acidic byproducts known to be deleterious to bioactive drugs and cells. 
By varying the ratio of components and copolymer composition or by modifying the polymer through chemical means, the properties of the carriers (including hydrophobicity and crystallinity) can change and adjust to present suitable degradation behavior and well-designed drug release.

Therefore, in the present study, we prepared MPEG- $b$-poly( $\varepsilon$-caprolactone-ran- $p$-dioxanone) (MPEG- $b$-PCDO) diblock copolymer arranged by 95:5 mol\% polyester block and examined the sol-to-gel phase transition at body temperature. Furthermore, we demonstrated the sustained release of protein drugs in vitro and in vivo using MPEG- $b$-PCDO copolymer hydrogels.

\section{Experimental Section}

\subsection{Materials}

MPEG (number-average molecular weight $\left.\left(M_{\mathrm{n}}=750 \mathrm{~g} / \mathrm{mol}\right)\right)$ and $\mathrm{HCl}(1.0 \mathrm{M}$ solution in diethyl ether), both from Sigma-Aldrich (Saint Louis, MO, USA), were used as received. CL was distilled over $\mathrm{CaH}_{2}$ under reduced pressure. DO (Metabio, Seoul, South Korea) was used as received. $\mathrm{CH}_{2} \mathrm{Cl}_{2}$ was distilled sequentially from $\mathrm{CaCl}_{2}$ and $\mathrm{CaH}_{2}$ under nitrogen before use. Pluronic F-127 (BASF, Ludwigshafen, Germany) is a polymer of PEO and PPO with two 100-unit hydrophilic PEO chains surrounding one 65-unit hydrophobic PPO chain and a total weight of $12,600 \mathrm{~g} / \mathrm{mol}$; it was used as received. Fluorescein isothiocyanate-labeled bovine serum albumin (BSA-FITC) and bovine-insulin (Zn salt, 28.8 IU/mg) were purchased from Sigma-Aldrich (Saint Louis, MO, USA).

\subsection{Characterization}

Proton nuclear magnetic resonance $\left({ }^{1} \mathrm{H}\right.$ NMR) spectra were measured using a Bruker $300-\mathrm{MHz}$ instrument (Billerica, MA, USA) with $\mathrm{CDCl}_{3}$ in the presence of tetramethylsilane (TMS) as the internal standard. Molecular-weight distributions of MPEG-polyester diblock copolymers were measured with an At-3000 GPC system (Shodex RI-71 detector; Futects, Seoul, South Korea) using two columns (Shodex K-802; Futects, Seoul, South Korea) and Shodex Asahipak GF-510; Futects, Seoul, South Korea). $\mathrm{CHCl}_{3}$ was used as the eluent at a flow rate of $0.6 \mathrm{~mL} / \mathrm{min}$. The viscosity of polymer solutions was measured using a viscometer (DV-III Ultra; Brookfield Engineering; Middleboro, MA, USA) with a programmable rheometer and circulating baths with a programmable controller (TC-502P). Viscosity measurements in the vessel were undertaken using a tight cap to prevent the evaporation of water in the polymer solution. The viscosity of polymer solutions was investigated using a T-F spindle at $0.2 \mathrm{rpm}$ from 6 to $70^{\circ} \mathrm{C}$ in increments of $1{ }^{\circ} \mathrm{C}$.

\subsection{Synthesis of MPEG-b-poly(E-caprolactone) (MPEG-b-PCL) and MPEG-b-poly(E-caprolactone- ran-p-dioxanone) (MPEG-b-PCDO) Diblock Copolymers}

$\operatorname{MPEG}\left(M_{\mathrm{n}}=750 \mathrm{~g} / \mathrm{mol}\right)(1.5 \mathrm{~g}, 2 \mathrm{mmol})$ and toluene $(30 \mathrm{~mL})$ were introduced into a flask. The MPEG solution underwent azeotropic distillation to remove water. Toluene was then distilled off completely. $\mathrm{CH}_{2} \mathrm{Cl}_{2}(91 \mathrm{~mL})$ was added to MPEG, followed by the addition of CL ( $\left.4.8 \mathrm{~g}, 42 \mathrm{mmol}\right)$ for MPEG- $b$-PCL, CL (4.8 g, $42 \mathrm{mmol})$ and DO (0.14 g, $1.7 \mathrm{mmol})$ for MPEG- $b$-PCDO, respectively. Polymerization was initiated by the addition of a $1.0 \mathrm{M}$ solution of $\mathrm{HCl}$ in diethyl ether $(4 \mathrm{~mL}$, $4 \mathrm{mmol}$ ) at $25^{\circ} \mathrm{C}$. After $24 \mathrm{~h}$, the reaction mixture was poured into $n$-hexane to precipitate a polymer, 
which was separated from the supernatant by decantation. The obtained polymer was re-dissolved in $\mathrm{CH}_{2} \mathrm{Cl}_{2}$ and filtered. The polymer solution was concentrated by a rotary evaporator and dried in vacuo to give a colorless polymer of quantitative yield. The MW of the PCL and poly(p-dioxanone) (PDO) segments in the diblock copolymer was determined by comparison of the intensity of the signal of the terminal methoxy proton of MPEG at $\delta=3.38 \mathrm{ppm}$, the signal of the methylene proton of PCL at $\delta=2.31 \mathrm{ppm}$ and the signals of the methylene protons of PDO at $\delta=4.15 \mathrm{ppm}$ in ${ }^{1} \mathrm{H}$ NMR spectroscopy.

\subsection{In Vitro Gel Formation and Protein Release Study}

Vials $(10 \mathrm{~mL})$ containing $5-\mathrm{mL}$ solutions of $20 \mathrm{wt} \%$ Pluronic F127, MPEG- $b$-PCL and MPEG- $b$-PCDO block copolymers in phosphate-buffered saline (PBS) were immersed in a water bath at $80{ }^{\circ} \mathrm{C}$ to dissolve the block copolymer above the melting temperature of PCL. Solutions were then left overnight at $4{ }^{\circ} \mathrm{C}$. For the protein-release experiment, $1 \mathrm{wt} \%$ BSA-FITC was added to the diblock copolymer solution at room temperature, and the mixture was mixed gently. A 1-mL aliquot of the diblock copolymer solution containing BSA-FITC was taken from the 5-mL solution, transferred to fresh $5-\mathrm{mL}$ vials and incubated at $37^{\circ} \mathrm{C}$ for $1 \mathrm{~h}$ to form a gel. Then, $4 \mathrm{~mL}$ of PBS at $37^{\circ} \mathrm{C}$ was added to each gel, and the vial was shaken at $100 \mathrm{rpm}$ at $37^{\circ} \mathrm{C}$. At specified sample collection times, $1 \mathrm{~mL}$ of solution was removed from the vial, and $1 \mathrm{~mL}$ of fresh PBS at $37{ }^{\circ} \mathrm{C}$ was added to the vial. Fluorescence spectroscopy (F-4500; Hitachi, Tokyo, Japan) was conducted immediately on the removed solution. The amount of cumulatively released BSA was calculated by comparison with a standard calibration curve prepared through measurements of solutions of BSA-FITC in PBS with various known concentrations. The released and remained amounts of BSA-FITC in block copolymer gels were individually calculated and compared with initial loaded BSA-FITC. The initial amount of loaded BSA-FITC showed almost similar amounts with the combined amount of released and remaining BSA-FITC. Three independent protein-release experiments were carried out for each gel composition.

\subsection{In Vivo Gel Formation and Protein Release Study}

To test the in vivo gel formation behavior of the MPEG- $b$-PCDO diblock copolymer, polymer solutions were injected into rats. Polymers were sterilized using ethylene oxide (EO) gas. Twelve Sprague-Dawley (SD) rats (320-350 g, 8 weeks), divided into three groups of four rats each, and were used in the release tests. The protocols of this study were approved by the Institutional Animal Experiment Committee at Ajou University School of Medicine (Gyeonggi-do, South Korea). The protocols of this study were carried out in accordance with the approved guidelines.

Three types of gel formulations were used in the in vivo release experiments: (i) a mixture of $1 \mathrm{~mL}$ of $20 \mathrm{wt} \%$ MPEG- $b$-PCL solution and $1 \mathrm{mg}$ of BSA-FITC or bovine-insulin; (ii) a mixture of $1 \mathrm{~mL}$ of $20 \mathrm{wt} \%$ MPEG- $b$-PCDO solution and $1 \mathrm{mg}$ of BSA-FITC or bovine-insulin; and (iii) a mixture of $1 \mathrm{~mL}$ of $20 \mathrm{wt} \%$ Pluronic F-127 solution and $1 \mathrm{mg}$ of BSA-FITC or bovine-insulin. Each gel formulation was prepared by dissolving the diblock copolymer in PBS and incubating at $4{ }^{\circ} \mathrm{C}$ for 1 day. Bovine-insulin was then added, resulting in the mixed solution. Within $5 \mathrm{~min}$ of the addition of bovine-insulin, a $1-\mathrm{cm}^{3}$ syringe with a $26-\mathrm{G}$ needle was used to inject $0.5 \mathrm{~mL}$ of the solution into the 
subcutaneous dorsum of a rat that had been anesthetized with ethyl ether. Resulting gels were used over 2 months for in vivo release experiments.

For the in vivo detection of BSA-FITC and bovine-insulin, an aliquot of blood was drawn from the tail vein of each rat at specified blood-collection times. A $0.3-\mathrm{mL}$ aliquot of blood from the catheterized tail vein was collected in an Eppendorf tube and mixed with $0.2 \mathrm{~mL}$ of a 1:499 mixture of heparin and saline, followed by vortex-mixing. To obtain plasma, the blood solution was centrifuged at $10,000 \mathrm{rpm}$ for $5 \mathrm{~min}$ at room temperature. Distilled water $(100 \mu \mathrm{L}), 66 \mathrm{mM}$ ethylene diamine tetra-acetic acid $(300 \mu \mathrm{L})$, and $50 \mathrm{mM}$ HEPES $(\mathrm{pH}$ 7.4) $(400 \mu \mathrm{L})$ were then added to the collected plasma. Plasma samples were stored at $-20^{\circ} \mathrm{C}$ until the assay.

To determine the reliable amount of BSA-FITC for each blood sample, we recorded the spectra of BSA-FITC standard solutions of known concentrations of BSA-FITC in blood. The measurement conditions were an excitation wavelength of $490 \mathrm{~nm}$ with a bandwidth at $2.5 \mathrm{~nm}$, an emission wavelength of $525 \mathrm{~nm}$ with a bandwidth at $2.5 \mathrm{~nm}$ and a response time of $2 \mathrm{~s}$. The amount of cumulatively released BSA was calculated by comparison with the standard calibration curves prepared with known concentrations of BSA-FITC. For each group of four rats treated with a particular gel formulation, the release experiment was separately performed on each rat, and the results were averaged.

For the bovine-insulin detection for each blood sample, the insulin concentration in plasma was determined using a bovine insulin enzyme-linked immunosorbent assay (ELISA) kit (Mercodia AB, Stockholm, Sweden). To run each assay, all reagents and samples were brought to room temperature. An aliquot from each sample $(25 \mu \mathrm{L})$ was transferred to a well plate strip. After following the manufacturer's instructions, absorbance was measured at $450 \mathrm{~nm}$ with an EL808 Ultra Microplate Reader (BioTek Instruments, Winooski, VT, USA). The amount of insulin released in vivo was calculated by comparison with standard calibration curves prepared with known concentrations of insulin using the bovine insulin ELISA kit. The area under the plasma insulin concentration versus time curve was calculated as the area under the curve (AUC).

\section{Results and Discussion}

\subsection{Synthesis of MPEG-b-PCL and MPEG-b-PCDO Diblock Copolymers}

MPEG- $b$-PCL and MPEG- $b$-PCDO diblock copolymers were synthesized by ring-opening polymerization (ROP) of CL and DO initiated by MPEG in the presence of $\mathrm{HCl}$ as the catalyst at room temperature. Typically, stannous octoate has been used for the conventional ROP of cyclic esters, such as CL, LA and GA. The ROP by stannous octoate carried out a reflux of high boiling point solvents, such as toluene. However, for biomedical applications, this organometallic catalyst and toluene are difficult to remove from the products, and the toxic contaminants remaining in the polymers may potentially affect the human body [26,27].

To solve these problems, our group has developed a metal-free ROP method using $\mathrm{HCl}$ as a monomer activator $[20,23,28]$. Preparation of MPEG- $b$-PCL and MPEG- $b$-PCDO diblock copolymers was accomplished by using an $\mathrm{HCl}$ catalyst at room temperature (Figure 1). The MPEG-polyesters diblock copolymers were obtained in high yields $(>95 \%)$ and showed narrow polydispersities 
(1.14-1.19) as measured by the GPC system. Based on our previous studies, we arranged the MW of MPEG- $b$-PCL diblock copolymers established at 750-2400 $\mathrm{g} / \mathrm{mol}$ to form thermo-responsive hydrogels at body temperature $[19,24]$. The unique MW of the MPEG- $b$-PCL and MPEG- $b$-PCDO diblock copolymers was optimized for the balance of amphiphilicity in an aqueous solution resulting in a thermosensitive sol-to-gel phase transition. The $5 \mathrm{~mol} \%$ portion of PDO added into the PCL main chains was determined to maintain the sol-to-gel transition at body temperature, because it is the gelation range at body temperature if these portions were increased. An MW ratio of 95:5 (PCL:PDO) in the polyester block was calculated by ${ }^{1} \mathrm{H}$ NMR and confirmed to be reasonable (Figure 2).

Figure 1. Synthetic scheme of methoxy poly(ethylene glycol) (MPEG) poly $\varepsilon$-caprolactone (PCL) (MPEG- $b$-PCL) and MPEG- $b$-poly( $\varepsilon$-caprolactone-ran- $p$-dioxanone) (MPEG- $b$-PCDO).

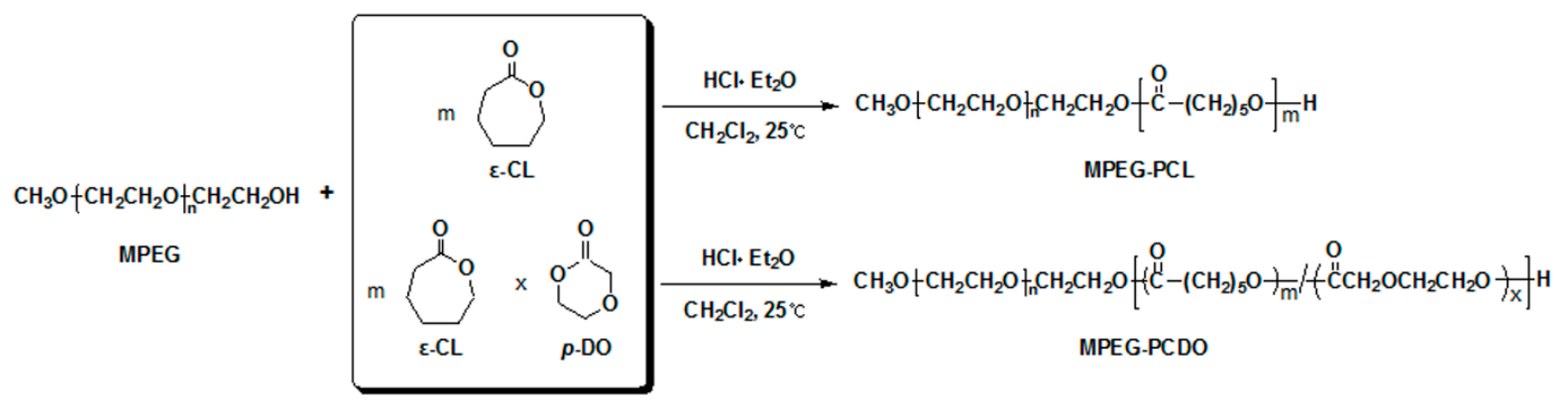

Figure 2. ${ }^{1} \mathrm{H}$ NMR spectra of (a) MPEG- $b$-PCL and (b) MPEG- $b$-PCDO in $\mathrm{CDCl}_{3}$.

\section{(a)}
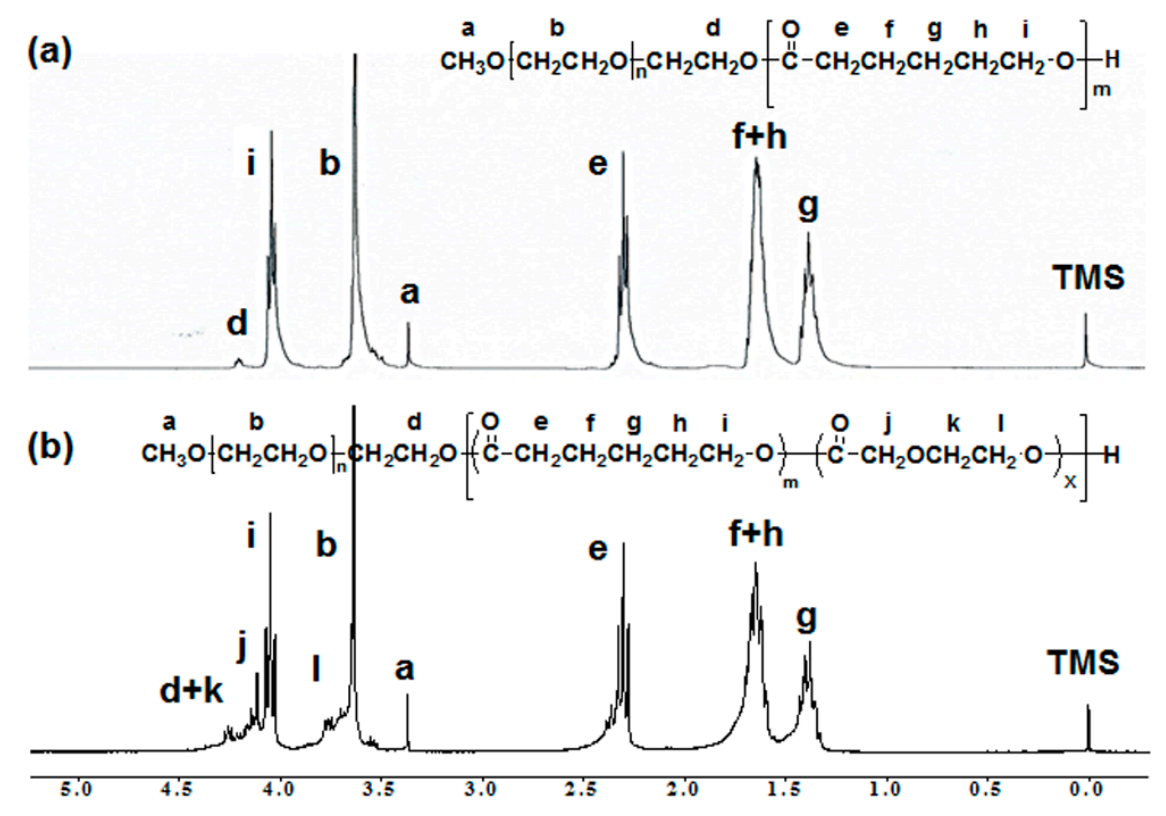

\subsection{Temperature- and Time-Dependent Changes in the Viscosity of MPEG-b-PCL and MPEG-b-PCDO Diblock Copolymers}

In order to ascertain the diblock copolymers' potential as injectable hydrogels, they were dispersed in aqueous solutions at $20 \mathrm{wt} \%$ concentrations to form translucent solutions at room temperature. In addition, the viscosities of the polymer solutions were measured with the gradual increase of temperature from 6 to $70{ }^{\circ} \mathrm{C}$. The $20 \mathrm{wt} \%$ concentration of diblock copolymers in aqueous solution 
was optimized for the sol-to-gel phase transition at body temperature, because the lower concentrations of diblock copolymers in aqueous solution showed gelation outside body temperature, while the higher concentrations had viscosities at room temperature.

The MPEG- $b$-PCDO solution started to form gels at approximately $30{ }^{\circ} \mathrm{C}$, while the MPEG- $b$-PCL solution began gelation at $27{ }^{\circ} \mathrm{C}$. These results suggested that the $5 \mathrm{~mol} \%$ portion of PDO mixed in $95 \mathrm{~mol} \%$ PCL chains contributed to the different temperature and range of gelation, as well as the different intensity of viscosity at $37{ }^{\circ} \mathrm{C}$. How the gelation of diblock copolymers responded to temperature changes is shown in Figure 3.

Figure 3. Temperature-dependent viscosity curves of Pluronic F127, MPEG- $b$-PCL and MPEG- $b$-PCDO diblock copolymers dissolved in $20 \mathrm{wt} \%$ aqueous solutions. The hydrogel shown in the photos was prepared from an MPEG- $b$-PCDO copolymer, and the gelation photo was taken immediately upon removal from a $37^{\circ} \mathrm{C}$ water bath.

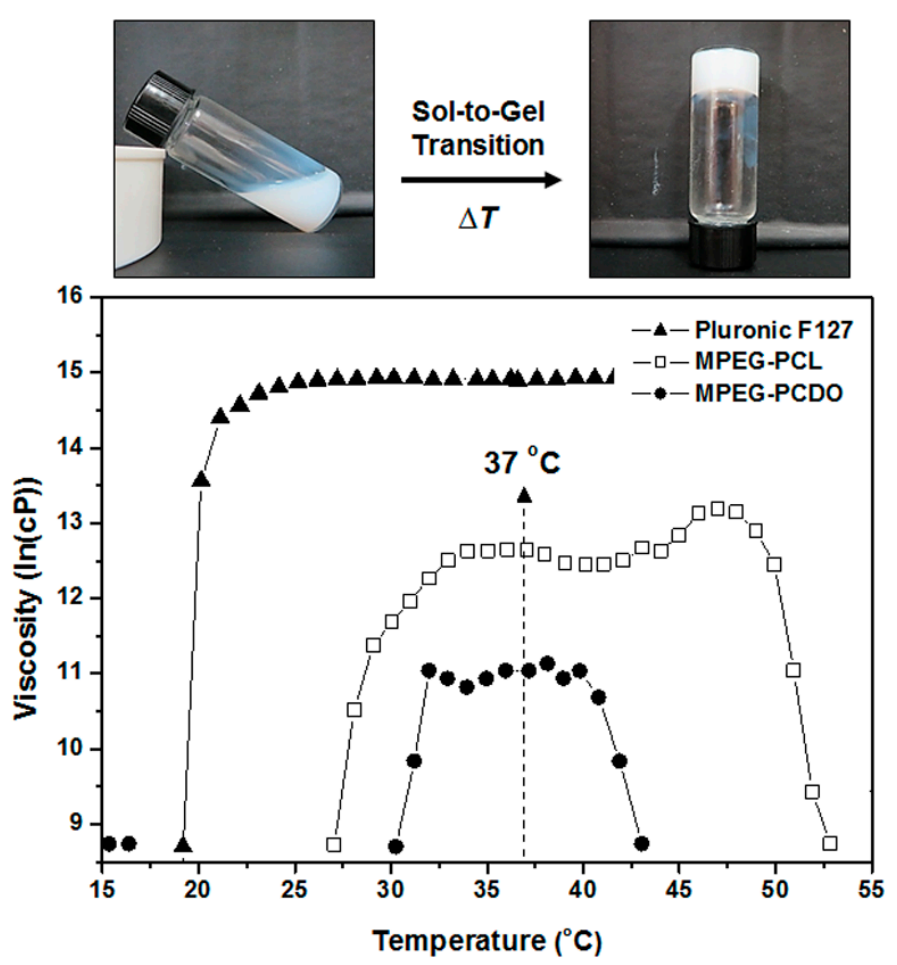

Furthermore, the hydrogels of MPEG-polyester diblock copolymers, including Pluronic F127, underwent time-dependent viscosity at $37{ }^{\circ} \mathrm{C}$ for $48 \mathrm{~h}$ (Figure 4). The viscosities of MPEG- $b$-PCL and MPEG- $b$-PCDO were increased and sustained over $40 \mathrm{~h}$, while the viscosity of Pluronic F127 decreased gradually after $15 \mathrm{~h}$. The slight increase of viscosity with time is generated by the hydrophobic interactions between PCL and PCDO chains in aqueous solutions, as reported in previous works $[19,20]$. The crystallization process of amphiphilic polymers lad to stronger packing interactions between the hydrophobic blocks, resulting in the increase of viscosity over time.

Although Pluronic F127 hydrogel shows a rapid sol-to-gel phase transition at body temperature, its use for the drug delivery system is limited due to the time-dependent gelation behavior. The hydrophobic interactions between PCL and PCDO chains in aqueous solutions were shown to 
gradually increase at body temperature. On the other hand, PPO units of Pluronic F127 hydrogel lead to weaker packing interactions between them, resulting in a gel-to-sol phase transition at a certain time.

Figure 4. Time-dependent viscosity changes of Pluronic F127, MPEG- $b$-PCL and MPEG- $b$-PCDO diblock copolymers solutions incubated at $37{ }^{\circ} \mathrm{C}$ for $48 \mathrm{~h}$. (a) MPEG- $b$-PCL; (b) MPEG- $b$-PCDO; and (c) Pluronic F127.

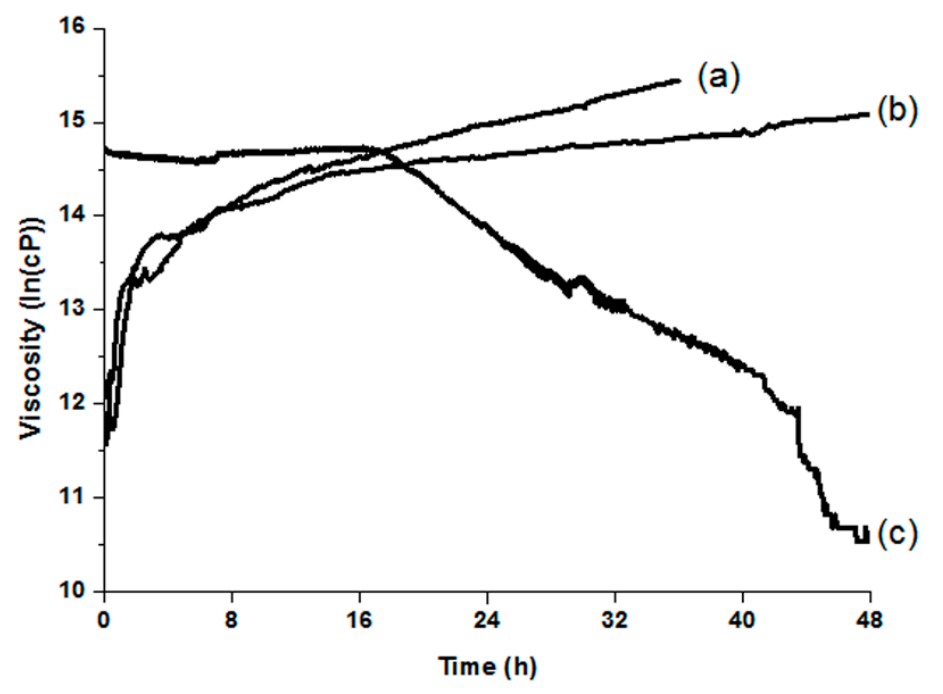

\subsection{In Vitro and in Vivo Release of Proteins from MPEG-b-PCL and MPEG-b-PCDO Hydrogels}

The 20-wt $\%$ solutions of MPEG- $b$-PCL and MPEG- $b$-PCDO copolymers and Pluronic F127 showed gelation at various intensities of viscosity at body temperature. Pluronic F127 has a 3-6-fold greater intensity of viscosity than that of the MPEG-polyester gels, but its viscosity is retained only for $15 \mathrm{~h}$, thus leading to the conclusion that it is not suitable for long-term drug delivery. When the 20 -wt $\%$ solutions of MPEG- $b$-PCL and MPEG- $b$-PCDO copolymers, as well as Pluronic F127 were injected subcutaneously into SD rats at room temperature, the copolymers became gels within a few seconds. Furthermore, the MPEG- $b$-PCL and MPEG- $b$-PCDO hydrogels retained their form for over a month at the injection site, whereas the Pluronic F127 gel dissipated 1-2 days post-injection.

For the in vitro drug release test, $20-\mathrm{wt} \%$ solutions of MPEG- $b$-PCL, MPEG- $b$-PCDO and Pluronic F127 were mixed with BSA-FITC at room temperature and incubated at $37{ }^{\circ} \mathrm{C}$ to form gels, followed by the addition of PBS ( $\mathrm{pH} 7.4$ ). BSA-FITC was quantitatively entrapped in MPEG- $b$-PCL, MPEG- $b$-PCDO and Pluronic F127. MPEG- $b$-PCL and MPEG- $b$-PCDO hydrogels were maintained in their initial shapes, and the BSA-FITC release was sustained over a period of time. However, the Pluronic F127 gel dissolved completely in PBS within $24 \mathrm{~h}$, resulting in an uncontrolled release of BSA-FITC. Figure 5 shows the cumulative amounts of BSA-FITC release from MPEG-polyester hydrogels and Pluronic F127 gel in various release patterns. The MPEG- $b$-PCL and MPEG- $b$-PCDO hydrogels, respectively, released BSA-FITC at approximately $25 \%$ and $45 \%$, for 15 days, whereas the Pluronic F127 hydrogel released over $90 \%$ of BSA-FITC within a day. As expected, the MPEG- $b$-PCDO showed a two-fold higher overall release compared to that of the MPEG- $b$-PCL hydrogel. This result indicates that the $5 \mathrm{~mol} \%$ of PDO block in PCL chains contributed to releasing more BSA-FITC from MPEG- $b$-PCDO hydrogel depending on the viscosity intensities at $37{ }^{\circ} \mathrm{C}$. 
The remaining BSA-FITC in hydrogels' post-release was confirmed by an excitation of the fluorescein using a blue light (465-490 nm), as shown in the photos of Figure 5.

Figure 5. In vitro release of BSA-FITC from gels prepared with $20-\mathrm{wt} \%$ solutions of MPEG- $b$-PCL and MPEG- $b$-PCDO diblock copolymers. The representative image of MPEG- $b$-PCDO hydrogel shown in the photo indicates the remaining BSA-FITC in the hydrogel three weeks post-release at $37^{\circ} \mathrm{C}$. The fluorescein in PBS solution ( $\left.\mathrm{pH} 7.4\right)$ excited by a blue light (465-490 $\mathrm{nm})$.

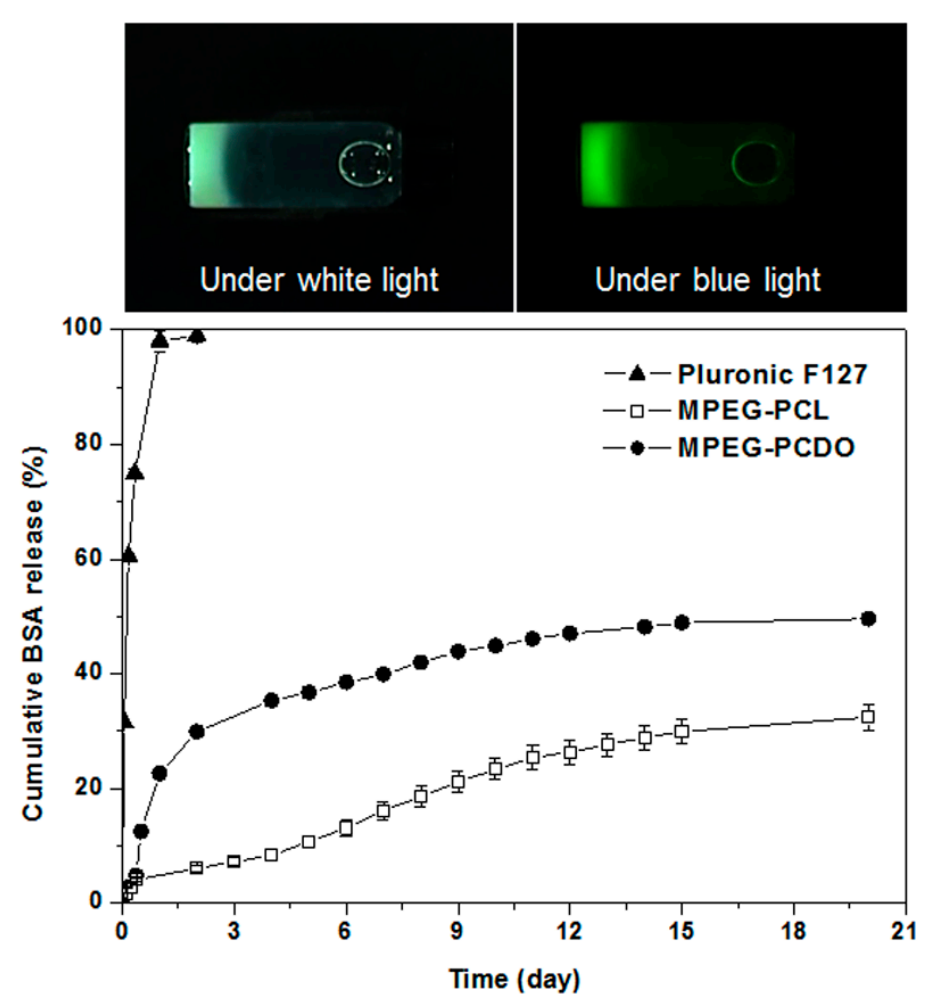

To verify the in vivo drug release behaviors of MPEG- $b$-PCL, MPEG- $b$-PCDO and Pluronic F127 hydrogels, BSA-FITC loaded hydrogels were injected subcutaneously into SD rats. Then, the blood collected from the tail vein at each time point was calculated for the released amounts of BSA-FITC. As shown in Figure 6, the in vivo release of BSA-FITC from the MPEG-b-PCDO hydrogel was observed only for one week, using a similar pattern as the previous MPEG- $b$-PCL hydrogel study [19]. This was due to the limitation of the fluorescein detected by a fluorometer depending on the fluorescein concentrations, even though the collected blood plasma contained the emitted BSA-FITC. The injection site in an SD rat was resected to confirm the remaining BSA-FITC in the hydrogel by using a blue light (465-490 $\mathrm{nm}$ ) exciting the fluorescein (see photos in Figure 6). The fluorescence image proves the continuous release of BSA-FITC from the hydrogel by showing the fluorescences on the inside of the skin around the hydrogel. 
Figure 6. In vivo release of BSA-FITC from injected hydrogels in SD rats $(n=4)$ for one weeks. The photo taken under white light shows an MPEG- $b$-PCDO hydrogel maintained at the injection site in a SD rat after one week. The fluorescence image under blue light (465-490 nm) indicates the remaining BSA-FITC in the hydrogel (arrowhead). * The in vivo result of BSA-FITC release from MPEG- $b$-PCL hydrogel is cited from a previous report [19].

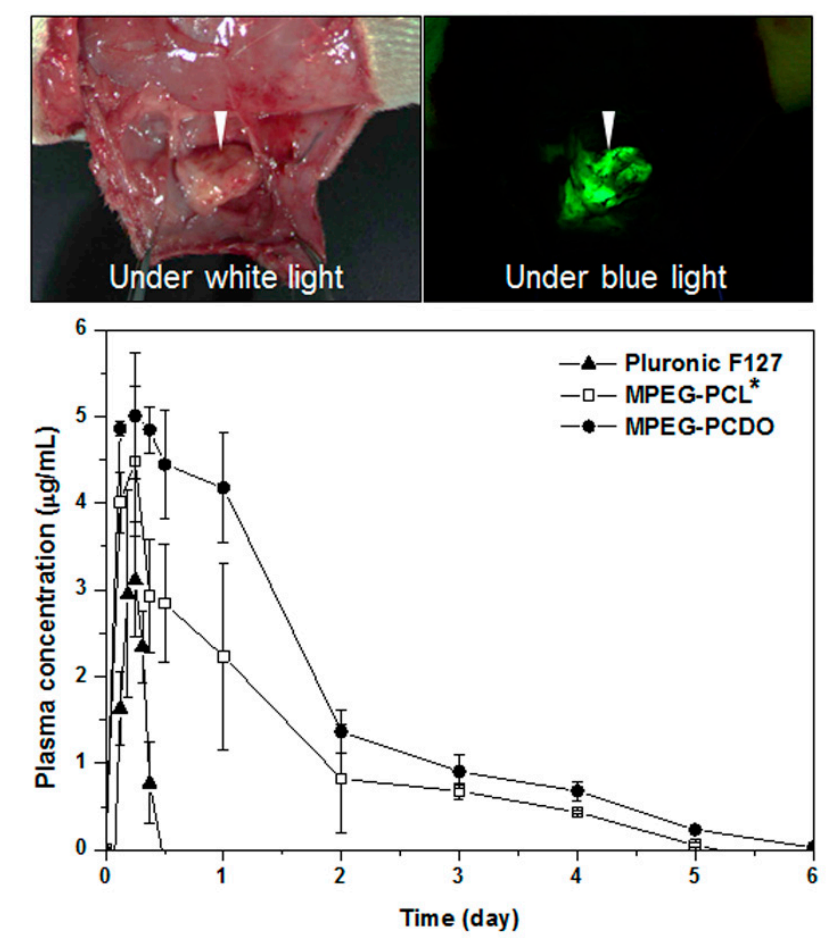

For a more accurate and reliable in vivo test of the model protein drug, bovine insulin was used for the long-term drug release in vivo, because even the smallest amount of this drug in the blood plasma can be detected by an ELISA method. As before, the 20 -wt $\%$ polymer solutions mixed with bovine insulin were injected into SD rats, and blood was collected from the tail vein at each time point to calculate the released amounts of bovine insulin. Insulin concentrations in blood released from MPEG- $b$-PCL and MPEG- $b$-PCDO hydrogels increased initially for one day and were sustained for 10 weeks, whereas the Pluronic F127 gel released a small amount of insulin for only two days (Figure 7). The $\mathrm{AUC}_{0-\mathrm{t}}$ values of MPEG- $b$-PCL and MPEG- $b$-PCDO hydrogels based on the release curves were $33.6 \pm 10.9$ and $82.3 \pm 7.8$, respectively, while the $\mathrm{AUC}_{0-\mathrm{t}}$ value of Pluronic F127 gel was only $3.91 \pm 0.53$. The MPEG- $b$-PCDO hydrogel showed a two-fold increase in the release of bovine insulin compared to that of the MPEG- $b$-PCL hydrogel for 10 weeks. This result corresponded with the in vitro release patterns of MPEG- $b$-PCL, MPEG- $b$-PCDO and Pluronic F127 hydrogels.

The Pluronic F127 hydrogel has high viscosity intensity at body temperature to prevent the initial burst of drug release, but the viscosity of Pluronic F127 hydrogel could not be maintained for over one day, as reported in previous work [19]. The $5 \mathrm{~mol} \%$ PDO chains distributed randomly in PCL chains enable not only enhanced release of drugs, but also rapid and durable gelation at body temperature. We achieved an enhanced and sustained release of protein drugs in vivo for 10 weeks using MPEG- $b$-PCDO hydrogel with an optimized $20-\mathrm{wt} \%$ concentration in aqueous solution. 
Figure 7. In vivo release of bovine insulin from injected hydrogels in SD rats $(n=4)$ for 10 weeks. (a) Release behaviors of Pluronic F127, MPEG- $b$-PCL and MPEG- $b$-PCDO hydrogels at full scale; (b,c) magnified curves depending on the release time and amount of bovine insulin.

(a) In Vivo Release of Insulin

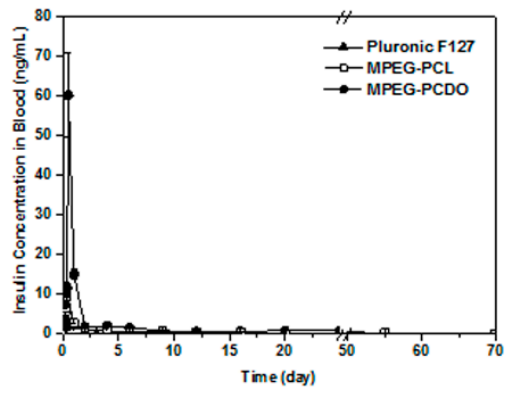

(b) Magnified Vertical Axis

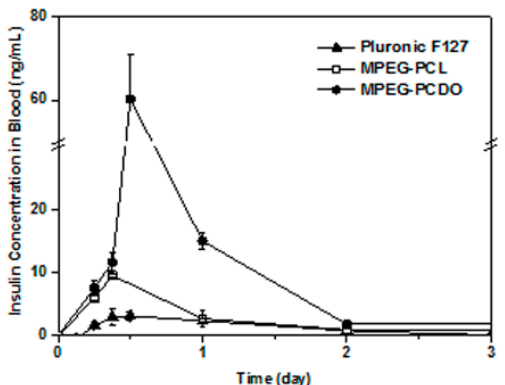

(c) Magnified Horizontal Axis

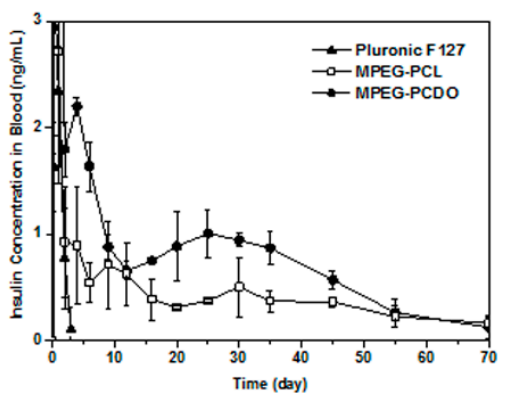

\section{Conclusions}

To obtain more controllable hydrogels in terms of the sustained delivery of drugs, we prepared a series of MPEG- $b$-PCDO diblock copolymers by ring-opening random copolymerization of 95:5 mol\% CL:DO with MPEG as the initiator in the presence of the $\mathrm{HCl}$ catalyst. The copolymers showed sol-to-gel transitions at body temperature when dispersed in aqueous solutions with an optimal $20 \mathrm{wt} \%$ concentration. Interestingly, the temperature range of gelation and viscosity intensity were changed by the addition of the PDO segment into PCL chains. At body temperature, the PDO units - components of MPEG- $b$-PCDO diblock copolymer distributed randomly in PCL chains - help to gelate and show a viscosity similar to that of MPEG- $b$-PCL hydrogels. The gelation property was consistently reflected in the release patterns of protein drugs in in vitro and in vivo experiments.

The in vitro release of BSA-FITC from MPEG- $b$-PCDO hydrogels showed a $45 \%$ release of BSA for 15 days, whereas the Pluronic F127 gel exhibited an almost complete release of BSA within a day. Furthermore, the sustained in vivo release of bovine insulin from MPEG- $b$-PCDO hydrogel was observed for 70 days. Therefore, MPEG- $b$-PCDO thermo-responsive hydrogels can be utilized for drug-delivery applications in terms of the specific gelation temperature, gel strength, degradation rate and controlled release of protein drugs.

\section{Acknowledgments}

This study was supported by a grant from Ministry of Trade, Industry and Energy (MOTIE) (Grant No. 10047811), a Basic Science Research Program (2013R1A2A2A04014200) and Basic Science 
Research Program through the National Research Foundation of Korea (NRF) funded by the Ministry of Education (NRF-2009-0093826).

\section{Author Contributions}

Designed the experiments: Moon Suk Kim; Performed the experiments: Hoon Hyun, Seung Hun Park and Doo Yeon Kwon; Analyzed the data: Gilson Khang, Hai Bang Lee and Moon Suk Kim; Wrote the paper: Hoon Hyun and Moon Suk Kim.

\section{Conflicts of Interest}

The authors declare no conflict of interest.

\section{References}

1. Kim, M.S.; Park, K. Injectable hydrogel. In Encyclopedia of Nanotechnology; Bhushan, B. Ed.; Springer: Dordrecht, The Netherland, 2012; Volume 2, pp. 1091-1096.

2. Aucoin, H.; Wilson, A.N.; Wilson, A.M.; Ishihara, K.; Guiseppi-Elie, A. Release of potassium ion and calcium ion from phosphorylcholine group bearing hydrogels. Polymers 2013, 5, 1241-1257.

3. Kim, J.I.; Kim, D.Y.; Kwon, D.Y.; Kang, H.J.; Kim, J.H.; Min, B.H.; Kim, M.S. An injectable biodegradable temperature-responsive gel with an adjustable persistence window. Biomaterials 2012, 33, 2823-2834.

4. Seo, H.W.; Kim, D.Y.; Kwon, D.Y.; Kwon, J.S.; Jin, L.M.; Lee, B.; Kim, J.H.; Min, B.H.; Kim, M.S. Injectable intratumoral hydrogel as 5-fluorouracil drug depot. Biomaterials 2013, 34, 2748-2757.

5. Peng, Y.; Li, J.; Li, J.; Fei, Y.; Dong, J.; Pan, W. Optimization of thermosensitive chitosan hydrogels for the sustained delivery of venlafaxine hydrochloride. Int. J. Pharm. 2013, 441, 482-490.

6. He, C.; Kim, S.W.; Lee, D.S. In situ gelling stimuli-sensitive block copolymer hydrogels for drug delivery. J. Control. Release 2008, 127, 189-207.

7. Agrawal, S.K.; Sanabria-DeLong, N.; Tew, G.N.; Bhatia, S.R. Structural characterization of PLA-PEO-PLA solutions and hydrogels: Crystalline vs. amorphous PLA domains. Macromolecules 2008, 41, 1774-1784.

8. Lee, J.; Bae, Y.H.; Sohn, Y.S.; Jeong, B. Thermogelling aqueous solutions of alternating multiblock copolymers of poly(L-lactic acid) and poly(ethylene glycol). Biomacromolecules 2006, 7, 1729-1734.

9. Kim, M.S.; Kim, J.H.; Min, B.H.; Chun, H.J.; Han, D.K.; Lee, H.B. Polymeric scaffolds for regenerative medicine. Polym. Rev. 2011, 51, 23-52.

10. Tsai, Y.; Jheng, L.; Hsu, S.L. Thermogelling polymers composed of poly (cyclohexylenedimethylene adipate) and poly(ethylene glycol). Eur. Polym. J. 2012, 48, 541-548.

11. Wang, F.; Shen, Y.; Xu, X.; Lv, L.; Li, Y.; Liu, J.; Li, M.; Guo, A.; Guo, S.; Jin, F. Selective tissue distribution and long circulation endowed by paclitaxel loaded PEGylated 
poly(e-caprolactone-co-L-lactide) micelles leading to improved anti-tumor effects and low systematic toxicity. Int. J. Pharm. 2013, 456, 101-112.

12. Kabanov, A.V.; Batrakova, E.V.; Alakhov, V.Y. Pluronic block copolymers as novel polymer therapeutics for drug and gene delivery. J. Control. Release 2002, 82, 189-212.

13. Lee, S.H.; Lee, J.E.; Baek, W.Y.; Lim, J.O. Regional delivery of vancomycin using pluronic F-127 to inhibit methicillin resistant Staphylococcus aureus (MRSA) growth in chronic otitis media in vitro and in vivo. J. Control. Release 2004, 96, 1-7.

14. Jeong, B.; Bae, Y.H.; Kim, S.W. In situ gelation of PEG-PLGA-PEG triblock copolymer aqueous solutions and degradation thereof. J. Biomed. Mater. Res. 2000, 50, 171-177.

15. Kim, M.S.; Hyun, H.; Khang, G.; Lee, H.B. Preparation of thermosensitive diblock copolymers consisting of MPEG and polyesters. Macromolecules 2006, 39, 3099-3102.

16. Jiang, Z.; Hao, J.; You, Y.; Gu, Q.; Cao, W.; Deng, Z. Biodegradable thermogelling hydrogel of P(CL-GL)-PEG-P(CL-GL) triblock copolymer: Degradation and drug release behavior. J. Pharm. Sci. 2009, 98, 2603-2610.

17. Wei, X.W.; Gong, C.Y.; Shi, S.; Fu, S.Z.; Men, K.; Zeng, S. Self-assembled honokiol-loaded micelles based on poly(caprolactone)-poly(ethylene glycol)-poly(caprolactone) copolymer. Int. J. Pharm. 2009, 369, 170-175.

18. Kang, K.N.; Kim, D.Y.; Yoon, S.M.; Lee, J.Y.; Lee, B.N.; Kwon, J.S.; Seo, H.W.; Lee, I.W.; Shin, H.C.; Kim, Y.M.; et al. Tissue engineered regeneration of completely transected spinal cord using human mesenchymal stem cells. Biomaterials 2012, 33, 4828-4835.

19. Hyun, H.; Kim, Y.H.; Song, I.B.; Lee, J.W.; Kim, M.S.; Khang, G.; Park, K.; Lee, H.B. In vitro and in vivo release of albumin using a biodegradable MPEG-PCL diblock copolymer as an in situ gel-forming carrier. Biomacromolecules 2007, 8, 1093-1100.

20. Kim, M.S.; Hyun, H.; Seo, K.S.; Cho, Y.H.; Lee, J.W.; Lee, C.R.; Khang, G.; Lee, H.B. Preparation and characterization of MPEG-PCL diblock copolymers with thermo-responsive sol-gel-sol phase transition. J. Polym. Sci. Part A 2006, 44, 5413-5423.

21. Cho, M.H.; Hyun, H.; Shin, Y.N.; Lee, J.W.; Lee, M.S.; Ahn, H.H.; Kim, M.S.; Lee, B.; Khang, G.; Lee, H.B. Release profile of BSA or BDNF from temperature sensitive hydrogels. Key Eng. Mat. 2007, 342-343, 473-476.

22. Kwon, J.S.; Yoon, S.M.; Shim, S.W.; Park, J.H.; Min, K.J.; Oh, H.J.; Kim, J.H.; Kim, Y.J.; Yoon, J.J.; Choi, B.H.; et al. Injectable extracellular matrix hydrogel developed using porcine articular cartilage. Int. J. Pharm. 2013, 454, 183-191.

23. Hyun, H.; Kim, M.S.; Khang, G.; Lee, H.B. Ring-opening polymerization of trimethylene carbonate by poly(ethylene glycol) in the presence of $\mathrm{HCl} \cdot \mathrm{Et}_{2} \mathrm{O}$ as a monomer activator. J. Polym. Sci. Part A 2006, 44, 4235-4241.

24. Kim, M.S.; Kim, S.K.; Kim, S.H.; Hyun, H.; Khang, G.; Lee, H.B. In vivo osteogenic differentiation of rat bone marrow stromal cells in thermosensitive MPEG-PCL diblock copolymer gels. Tissue Eng. 2006, 12, 2863-2873.

25. Shim, S.W.; Kwon, D.Y.; Park, J.H.; Kim, J.H.; Chun, H.J.; Koh, Y.J.; Kim, M.S. Preparation of zwitterionic sulfobetaine end-functionalized poly(ethylene glycol)- $b$-poly(caprolactone) diblock copolymers and examination of their thermogelling properties. J. Polym. Sci. A 2014, 52, $2185-2191$. 
26. Davies, A.G. Organotin Chemistry, 2nd ed; Wiley-VCH: Weinheim, Germany, 2004.

27. Pitt, C.G.; Schindler, A. In Long-Acting Contraceptive Delivery Systems; Zatuchni, G.L., Goldsmith, A., Shelton, J.D., Sciarra, J.J., Eds.; Harper and Row Publishers: Philadelphia, PA, USA, 1984; pp. 48-63.

28. Kim, M.S.; Seo, K.S.; Khang, G.; Lee, H.B. Ring-opening polymerization of $\varepsilon$-caprolactone by poly(ethylene glycol) by an activated monomer mechanism. Macromol. Rapid Commun. 2005, 26, 643-648.

(C) 2014 by the authors; licensee MDPI, Basel, Switzerland. This article is an open access article distributed under the terms and conditions of the Creative Commons Attribution license (http://creativecommons.org/licenses/by/4.0/). 